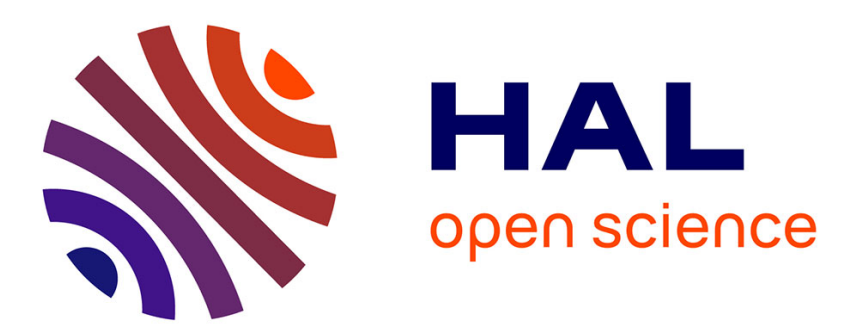

\title{
Phase-shifting digital speckle pattern interferometry: off-the-shelf setup description and application to stainless steel membrane displacement measurements
}

Pierre Slangen, B. Gautier

\section{- To cite this version:}

Pierre Slangen, B. Gautier. Phase-shifting digital speckle pattern interferometry: off-the-shelf setup description and application to stainless steel membrane displacement measurements. Speckle Metrology 2003, Jun 2003, Trondheim, Norway. pp.76, 10.1117/12.516580 . hal-02012250

\author{
HAL Id: hal-02012250 \\ https://hal.science/hal-02012250
}

Submitted on 8 Jun 2021

HAL is a multi-disciplinary open access archive for the deposit and dissemination of scientific research documents, whether they are published or not. The documents may come from teaching and research institutions in France or abroad, or from public or private research centers.
L'archive ouverte pluridisciplinaire HAL, est destinée au dépôt et à la diffusion de documents scientifiques de niveau recherche, publiés ou non, émanant des établissements d'enseignement et de recherche français ou étrangers, des laboratoires publics ou privés. 


\title{
Phase Shifting Digital Speckle Pattern Interferometry : Off-the-shelf set-up description and application to stainless steel membrane displacement measurements.
}

\author{
P. Slangen, B. Gautier, \\ Ales School of Mines, Dpt CMGD-MMS, F-30319 ALES CEDEX France
}

\begin{abstract}
Phase shifting digital speckle pattern interferometry (PDSPI) is well suited for micrometric displacement measurements. It is non-intrusive and without contact for the object under investigation. Speckle is generated when a beam of coherent light impinges the surface of an optically rough object.

Designing the system with off-the-shelf components implies the full understanding of speckle generation, image acquisition and processing. This paper will describe the main components and their contribution to the final result, from the speckle effect to the unwrapping of the phase.

The designed PSDSPI set-up allows the study from small to large areas and can produce quantitative displacement maps using phase shifting principle. As an application the object is a flat circular steel membrane closing a depression chamber. The size of the membrane is about $20 \mathrm{~mm}$ in diameter. The membrane deformation is measured using step by step PSDSPI process while decreasing pressure in the chamber. The results show a typical displacement of about $1 \mu \mathrm{m}$ for 1 mbar. Quantitative profiles of the membrane deformation can be obtained.

Further applications will be presented, including measurement corrections by shape of the object. Phase discontinuities will be discussed.
\end{abstract}

Keywords: Speckle, Interferometry, Phase Shifting

\section{INTRODUCTION}

Holographic and Speckle methods are used for displacement or deformation measurements because they are nonintrusive and no contact. The spatial resolution of the data field is very high and can be fully obtained in one measurement. The phase variation of the object under investigation is determined by computing different images. These images can be acquired in real-time if the object deformation is continuous or step by step if the object undergoes different states of deformation. The processing of the images to retrieve displacement information can also be done in real time or post processed. This is typically dependant on the computer processor and the on the phase computation algorithm (2, 3, or more phase shifts). The number and the kind of phase shift also strongly affect the error on the phase and sometimes require strong hypotheses. Finally for some extreme cases the illumination and observation geometry and even the object shape can generate huge errors.

All these parameters must be taken into account while buying an off-the-shelf PSDSPI set-up or during the construction of such an interferometer with off-the-shelf and "home-made" parts. Moreover a lot of users would prefer open-ended software for either the acquisition or processing of the images or the management of the results. As an extensive "systematic approach to TV Holography" has been published by Doval 1, we will focus on the applied conditions required for the PSDSPI set-up developed by our lab. Results for the displacement measurement of a plane membrane will be shown. Suggestions will be done about the phase discontinuities appearing for some wrapped phase map.

\section{DESCRIPTION OF THE SET-UP}

The PSDSPI set-up also includes a 2W CW Verdi laser emitting at 532nm. PSDSPI system can be split in image acquisition and processing. We will describe the optical components and the software separately, even if there are often linked together. Sometimes real time processing requires a smart combination of processing and hardware configuration. Real time acquisition routine can also be used for static recording.

In the developed set-up, moving a mirror stuck on a piezoelectric transducer (PZT) located in the reference arm generates phase shift. The reference beam impinges the CCD sensor through optical lenses or from an optical fiber. The optical fiber configuration is more convenient to move the camera where it is required, but the fiber must be isolated from external perturbation (bending, temperature variation, mechanical efforts, ...) to avoid drift. The object beam 
propagates through the set-up via mirrors and lenses to impinge on the object with desired angle of illumination. We can recall here that the sensitivity of the phase to displacement belongs to the bisector of illumination and observation directions. The measurements have been done with pure out of plane sensitivity. Therefore the observation and illumination directions are parallel and strike the object surface orthogonally. The illumination beam is collimated to ensure equal sensitivity across the diameter of the membrane. Because the surface is flat, no shape compensation has been achieved.

\section{Acquisition}

The optical components and other electronic devices (PZT, CCD camera, ...) are located on a single plate allowing the transport of the whole set-up, including the laser source. The interferometer is out of plane sensitive, i.e. the reference beam illuminates the CCD while the object beam illuminates the object to produce subjective speckle. Subjective speckle is generated when the coherent light diffused by the object passes through the photographic objective (lenses and aperture) of the CCD camera. For a given wavelength, the subjective speckle size is fixed by the focal length p' and the aperture diameter $D$ (Fig. 1 ): $d_{s}=\lambda$ p'/D

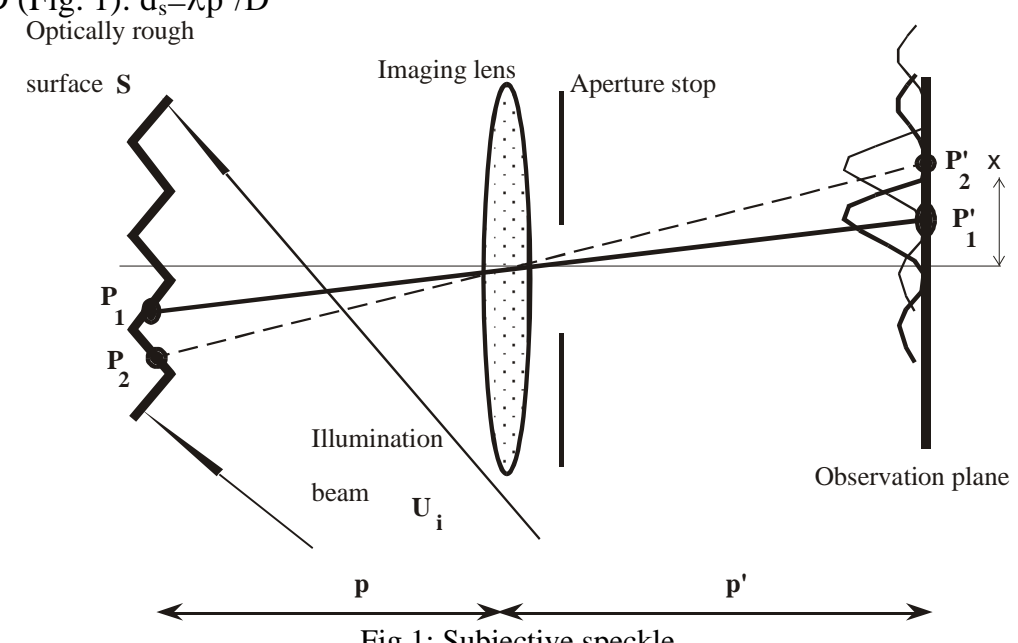

Fig.1: Subjective speckle

When needed, optics are coated to match the laser wavelength. This avoids secondary reflections and the generation of undesirable fringes. The laser beam is split in reference and object beams by a prism to ensure the maximal power in the object beam. The reference path is not matched to the object path because the laser is highly coherent $\left(\mathrm{L}_{C}>30 \mathrm{~m}\right)$.

\subsection{Phase shifting}

Temporal or spatial phase shift can be used. Phase shift is temporal when the phase is shifted in time while it is spatial when the phase is shifted across the CCD. Spatial phase shifting is more convenient for fast acquisition but requires a lot of CCD spatial resolution. Temporal phase shifting can even be used for very fast acquisition and works full resolution ${ }^{2}$. To compute the phase of the object, phase shifting is produced by the PZT to displace the reference mirror. The commercial PZT can deliver a $0->3 \mu \mathrm{m}$ displacement versus $0->1000 \mathrm{~V}$ voltage. The voltage is generated by a Digital to Analog Converter. The DAC is computer-controlled via serial bus. The calibration of the PZT has been achieved by adding a Michelson interferometer in the reference arm ${ }^{3}$. It is also possible to determine the "PZT voltage versus phase shift" function by measuring the light intensity at the exit of the reference optical fiber (Fig.2).

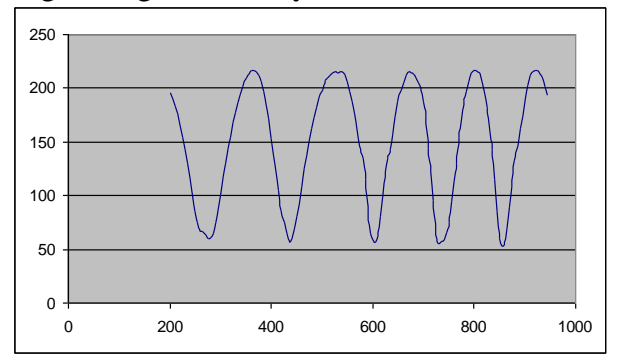

Fig.2: PZT voltage (Digital Volt) versus phase change curve (gray level)

This assumes the injection of the two Michelson beams in the fiber core. After calibration the "four buckets" procedure is then completed to get four phase shifted images allowing the phase calculation. The "four buckets" is used because it is a good balance between accuracy and the number of images ${ }^{4}$. Some authors are using only 2 phase shifts but this 
implies strong conditions such as background and modulation intensities being constant during the measurement 5 . Temporal speckle pattern is also produced with continual deformation of the object ${ }^{6}$. Comparing the phases of an object before and after deformation also implies the phase stability of the set-up. Phase drift measurements have been done and show good stability of the fringes (drift about $\lambda / 2000$ by second in a "cool" place). Random phase drift can appear when the set-up is under industrial environment but some authors have reported solutions to overcome it ${ }^{7}$.

\subsection{Interferometric head}

This head has been manufactured in our lab to cope with the C-mount of the CCD camera and standard photographic objectives. Moreover, the generated spacing enables to place the beam mixing plate. Mixing the object beam and the smooth reference beam produces the interference on the CCD (specklegram). Fig.3 shows the head with "open beam" passing through convergent lens to expand on the CCD chip area. For fiber illumination the lens holder is replaced by the fiber chuck holder.

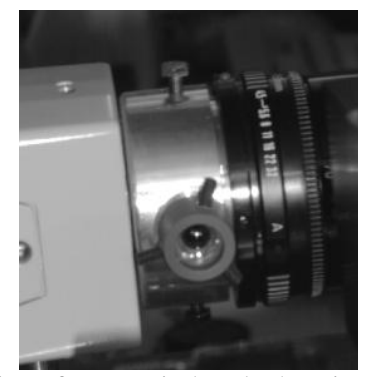

Fig.3: The interferometric head, showing lens holder

\subsection{CCD Camera}

The CCD camera has an $800 * 600$ pixels resolution and $25 \mathrm{~Hz}$ frame rate ( $1 / 2$ inch sensor, Interline, 8 bits). Settings of the camera, such as gain, shutter speed, white and black levels, are computer-controlled via RS485 serial bus. The main advantages of the camera are the high sensitivity (about 0.1 lux) and very fast shutter speed (1/1000000 s). The frame grabber connected to the camera can record 8 bit images at $25 \mathrm{~Hz}$ full resolution. Settings of the board can also be managed by the user. It is very important to control these devices to get the best image contrast. This ensures to easily set a good balance between reference and object beams intensities. This ratio determines the contrast of the final fringes, even if the phase shift processing already enhances it. Moreover, good settings also avoid too much bad pixels. Bad pixel occurs when the gray level of a pixel is much different than the pixels in a user-defined neighborhood.

\subsection{Acquisition software}

The acquisition software is made of several "virtual instruments" (or subroutines) developed under LabView (National Instruments). It is then possible to simultaneously drive all the parts of the set-up from a distant room and to delay all the measurement procedures if required. A laser driver has also been developed. It is then possible to program long time measurement without the mandatory presence of the user. This has been very valuable when applied to measurement on drying concrete (about 6 hours measurement) or when the laser supply and computer fans produces too much optical noise for very sensitive experiments.

The acquisition requires 4 phase-shifted images. Calibration of the PZT has been achieved before experiment. This ensures the PZT to produce $0, \pi / 2, \pi$ and $3 \pi / 2$ phase shifts before each camera acquisition. One set of four images is recorded within a second. This is mainly due to the PZT device that requires 3 commands to be at the right phase shift. The images are then stored in memory or on the hard disk for further processing.

\section{Processing of the specklegrams}

\subsection{Subtraction}

Specklegrams can be processed in "real time" or post processed to retrieve phase difference information. The four buckets algorithm computes the phase of each state. A mask of modulation is also determined by comparing the modulation intensity to a user-defined threshold intensity. This mask allows the localization of the object in the image and also the possibility to automatically detect holes in the scene. Subtraction of the different states yields to the typical saw-teeth fringe pattern (wrapped fringes). Modulation masks of the phase maps are combined to give the subtraction mask. Wrapped fringes appear because the phase is computed between $[0,2 \pi]$ and subtraction gives a final $[0,2 \pi]$ image.

\subsection{Fringe Unwrapping}

Fringe unwrapping consist in retrieving and removing the $2 \pi$ steps. The software runs a C++ program under LabView by calling a "Code Interface Node". This allows the combinaison of the nice LV user interface and the computation power of $\mathrm{C}++$. The algorithm has been published by the author ${ }^{8}$ and is called "DIsk GROwing Algorithm" (DIGRO). 
The basic principle of the program is to propagate from a point of the image by generating disks from this point. The starting point is mouse-defined or is the center of the image. The program then searches for the best point in this area to start the first disk. The center of the first disk must be a point with low noise; that is the phase variation in the neighborhood of this point must vary slowly. After the first disk, secondary disks are growing from the perimeter of the first one (like Huygens principle for wave propagation). This kind of propagation allows coming back and to go round bad pixels clusters. Moreover pixels are unwrapped only if the gray level is within an accuracy range compared to the phase of the pixel being unwrapped. That is if the pixel under unwrapping is in a bad accuracy cluster (phase difference variation very noisy) the pixel is not unwrapped. During the propagation an unwrapping mask is also generated. To be unwrapped a pixel must have at least 2 neighbors unwrapped in a $3 \times 3$ cluster. Of course to retrieve phase steps, multiples of $\pi$ are added or subtracted to enhance or to reduce the phase difference value and so to lead to the unwrapped image. The continuous phase map is then shown and the unwrapping mask can be used for further processing such as least square filtering to force a value for bad pixels (if needed).

Finally the geometry of the set-up is taken into account and a false color 3D plot is presented for the displacement map. The main advantage of this algorithm is that it is possible to propagate even if the fringes are very noisy and to get a continuous profile within defined accuracy $(\lambda / 32 \mathrm{max})$. The time to unwrap an $800 * 600$ pixels image is about 5 seconds or less, regarding accuracy, noise and fringe distribution (for standard PC, Pentium III 500MHz). Anyway DIGRO must be adapted to cope with phase discontinuities, e. g. when using this algorithm in fringe projection to get object shape.

\subsection{Errors on phase difference measurements}

The different parts of the set-up (PZT calibration, CCD noise, four buckets algorithm, phase unwrapping, phase drift) contribute to the error on the phase measurement. Final tests have been carried on reference object. This object is undisturbed and not temperature sensitive. Subtraction of phase maps obtained for the same rest state is done. In this case the phase difference must be zero. But the errors from the different parts generate a distribution of the phase difference around zero. For a "cool" place, the half bandwidth is about $\lambda / 13$.

\section{APPLICATION}

The developed set-up has been successfully applied to the measurement of displacement of a metallic membrane. The metallic membrane has a $20 \mathrm{~mm}$ diameter and is about $1 \mathrm{~mm}$ thick. It is fixed with 6 screws on the body cavity. A screw pump manages the pressure in the cavity (Fig.4).

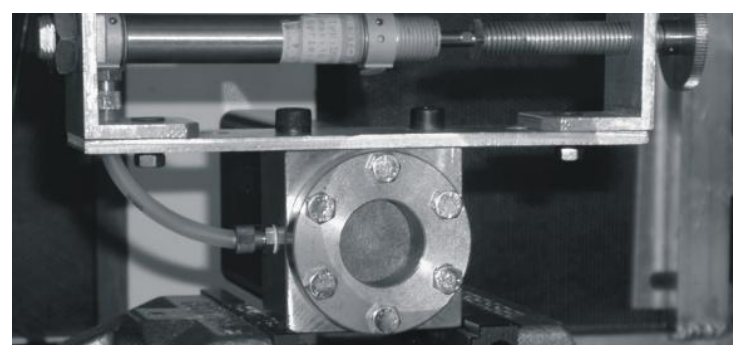

Fig.4 : The membrane under laser illumination, screw pump on top

The pressure is measured by a digital pressuremeter. No special diffusing paint is needed and the subjective speckle is generated under laser illumination of the optically rough surface imaged by a standard photographic zoom lens. Fig.5 presents a typical wrapped image for a 1 mbar pressure variation.

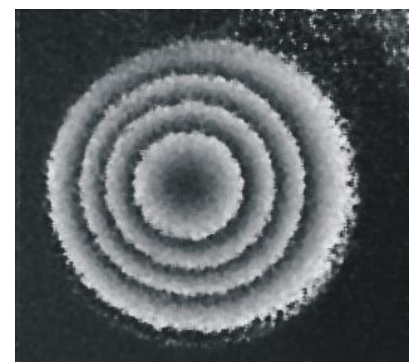

Fig.5 : Wrapped phase difference map for 1 mbar pressure variation 
Unwrapping of these fringes leads to 3D plot and Z- profile of the membrane allowing the full displacements map characterization without any contact. The displacement generated by the pressure difference is about $1 \mu \mathrm{m}$ (Fig.6)
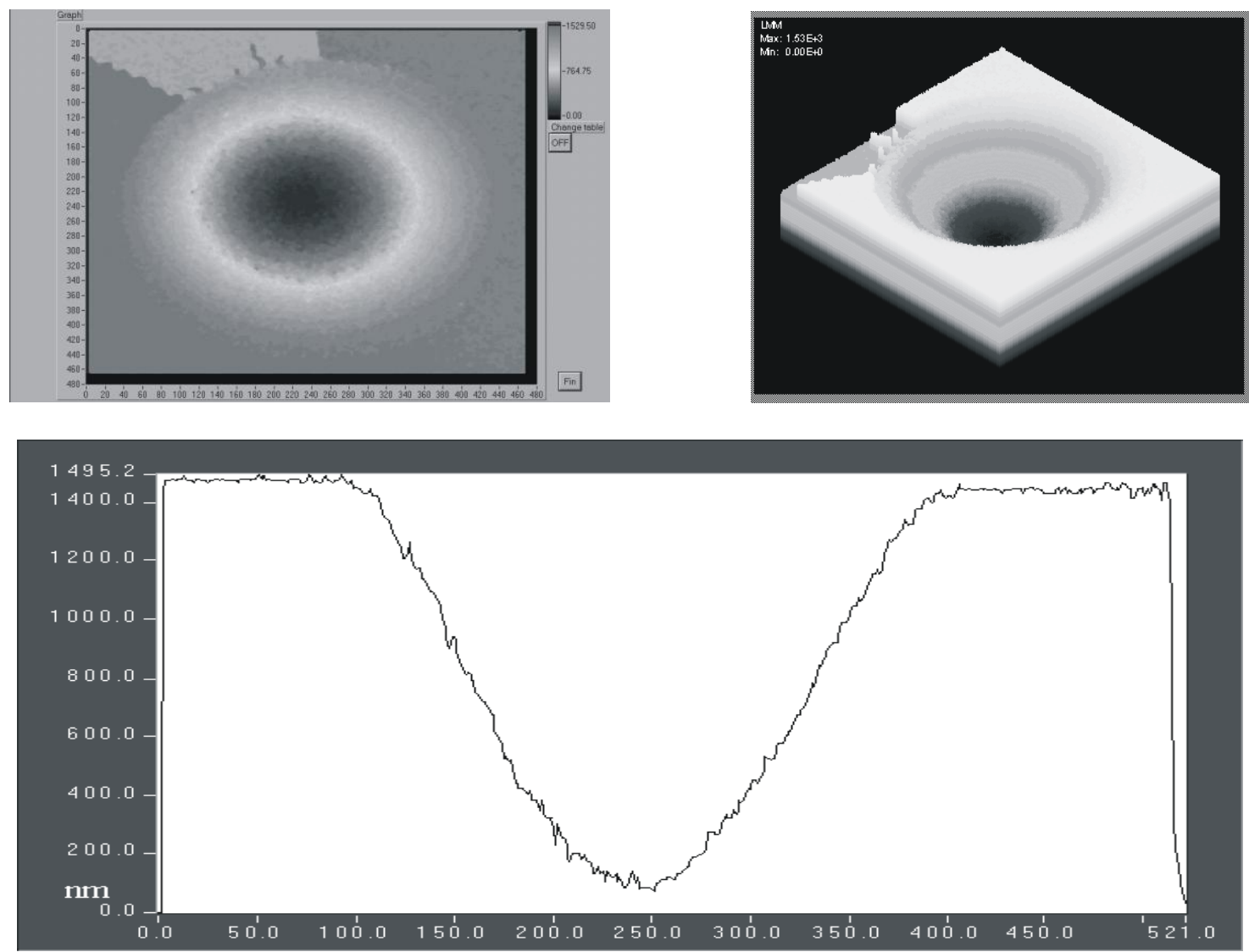

Fig.6 : Continuous phase map, 3D image and diagonal $\mathrm{Z}$ profile $(\mathrm{nm})$ of the membrane

\section{DISCUSSION}

While building or when buying a PSDSPI interferometer, the final user must take into account a lot of problems. If the application must be real-time, focus must be done on the phase shifting device and the number of images to collect. The size of the object is also drastically affecting the set-up because it requires huge laser power or to pulse the laser source. For the last, synchronization between the camera and the laser pulse should be achieved. At the end of the process it is more and more required to give a measurement uncertainty for the final quantitative measurement. The contribution of the different parts of the set-up must then be determined to qualify the displacement. This can be done part by part or for the whole entity. For large objects it is often not possible to illuminate with collimated beams. In this case the change of sensitivity vectors across the surface object may produce errors in measurement close to $100 \%$. The same problem occurs if small non-flat object is under investigation. Then it is the projection of the sensitivity vector in the object plane that is not often obvious.

During this work we also applied the phase unwrapping algorithm to phase maps presenting phase discontinuities. The modification of the DIGRO algorithm is now under development to cope with such discontinuities.

We can point out the development of the PSDSPI and other optical methods in the field of non-destructive testing and mechanical studies. Years ago it was already nice to record and process 4 images in the row. Now it is possible to use very fast acquisition rates (about $1 \mathrm{kHz}$ ) and computing processor at $2 \mathrm{GHz}$ with live memory. Speckle was first considered as noise in holographic fringes. Then it was the information carrier for speckle photography, and generates the holes pair producing Young's fringes. Speckle techniques are now split in speckle photography and speckle interferometry. 
From speckle photography, techniques such digital image correlation are now used to perform in plane displacement measurement. If the 3D components are useful, stereocorrelation can now be used. The advantage of which is the possibility to adjust the resolution by changing the optical magnification.

In speckle interferometry and also digital holography, the information carrier is the computed phase map. The resolution is some nanometers whatever the investigated area. Nowadays it is technically easy to compute such a phase map. Assuming that phase discontinuities can be considered as noise for now, we are sure that the phase discontinuities will act as an information carrier in the future. On the eve of this $21^{\text {st }}$ century, the study of digital phase generation, distribution, and evolution will probably request as much reflection and scientific works as laser speckle did during the last years.

\section{CONCLUSION}

In this paper we present the key points allowing the final user to buy or to build a Phase Shifting Speckle Interferometer, from the speckle effect to the phase unwrapping, including phase to displacement conversion.

Contribution of the different parts of the set-up and their contribution to the final phase difference measurement have been shown. Application to the displacement of a steel membrane has been carried out. Results, as displacement maps, present a final uncertainty of measurement about $\lambda / 13$ at $532 \mathrm{~nm}$.

\section{REFERENCES}

[1] A.F. Doval, “A systematic approach to TV holography”, Meas. Sci. Technol., Vol.11, R1-R36, 2000.

[2] P. Jacquot, V. Chalvidan, "Interferometrie speckle a $1 \mathrm{kHZ}$ en laser pulse", Acte Methodes et techniques optiques pour l'Industrie, Vol.1, pp. 135-142, CMOI SFO, Tregastel (France), 2001.

[3] P. Slangen, C. De Veuster, Y. Renotte, L. Berwart, Y.Lion,"Computer-aided interferometric measurements of drift and phase shifter calibration for DSPI", Opt. Eng., Vol.34 (12), pp 3526-3530, Dec 1995.

[4] K. Creath, "Phase-shifting Holographic Interferometry" in Holographic interferometry. Principles and methods, P.K. Rastogi, Springer-Verlag, chapter 5, pp 109-150, Berlin, 1994.

[5] Sesselmann M.; Albertazzi Goncalves A., "Single phase-step algorithm for phase difference measurement using ESPI”, Proc. SPIE, , Laser Interferometry IX: Techniques and Analysis, Vol. 3478, pp. 153-159, Malgorzata Kujawinska; Gordon M. Brown; Mitsuo Takeda; Eds., 6/1998.

[6] Li Xide; Yang Yizhang; Tao Gang; Li Rongxian, "Deformation analysis with temporal speckle pattern interferometry", Opt. Eng., Vol.40 (02), pp.310-317, 2/2001.

[7 ] D. Findeis, D. R. Rowland, J. Gryzagoridis, , "Vibration Isolation techniques suitable for portable Electronic Speckle Pattern Interferometry", Proc. NDE Workshop, Non Destructive Evaluation of High Valued Engineering Systems, ARO-FE, Cape Town, April 2002.

[8] C. De Veuster, P. Slangen, Y. Renotte, L. Berwart, Y.Lion, ""Disk Growing" algorithm for phase-unwrapping: Application to speckle interferograms", Appl. Opt., Vol.34 (2), pp 240-247, Jan. 1996. 